\title{
Recall of paired-associate items as a function of interpolated pairs of different types
}

\author{
WILLIAM J. THOMSON \\ STANFORD UNIVERSITY, STANFORD, CALIFORNIA
}

A continuous presentation task was used to study interference effects of well-known and unknown items. It was found that insertion of well-known items between study and test had little effect on recall, while insertion of unknown items considerably reduced recall. The results were. interpreted in terms of a mathematical model of memory.

A recent paper by Atkinson \& Shiffrin(1967) describes the application of a multiprocess model of memory to an array of experiments on memory and learning. The principal component of the model is a rehearsal buffer in which new items to be learned are processed and transcribed into a long-term store. One experiment described in their paper concerns the effects of various rehearsal schemes on the buffer. The experiment utilized a continuous presentation paired-associate task similar to that employed in the present paper, and Ss were instructed to rehearse items either overtly (by pronouncing aloud) or covertly. In a number of analyses it was found that overt rehearsal led to higher recall than covert rehearsal, and this result was explained in terms of the buffer model. The model assumes that items to be learned have some probability, $\alpha$, of entering the buffer. With overt rehearsal it was assumed that an item always entered the buffer $(\alpha=1)$, whereas with covert rehearsal an item was less likely to enter the buffer. Using these restrictions on $\alpha$, curves generated by the model flt the data quite well. These fits were interpreted to imply that the overt manipulation was successful in increasing to near unity the probability of an item entering the buffer. The present study deals with procedures which reduce this probabllity to near zero for some items. The experimental manipulation involves the interspersing of well-known items into a continuous list of unfamiliar items.

wethed

Subjects. The Ss were eight Stanford University students. Each $\mathbf{S}$ participated in 10 experimental sessions and was paid two dollars per session.

Apparatus. All portions of the experiment were programmed and run by a PDP-1 computer under the control of a time-sharing system. Stimuli were displayed on the face of a cathode-ray tube (CRT), and responses were made on an electric typewriter keyboard directly below the CRT. For a more detailed description of the apparatus, see Atkinson, Brelsford, \& Shiffrin (1967).

Stimuli and responses. A stimulus-response pair will be referred to as an item. The stimulus portion of an item consisted of one of 216 low-association CVC trigrams, and the response portion of an item was a digit from 0 to 9 . For each $\mathrm{S}$ two CVC trigram-response pairs were designated as known (k) items. A kitem remained the same for an $\mathrm{S}$ throughout all 10 sessions. The remaining 200 trigram-response pairs were designated as unknown (u) items. A u item was presented only once in a session with its response member being assigned at random.

Procedure. Each session began with six practice study trials during which a CVC and its response appeared on the CRT for $2 \mathrm{sec}$. After the initial study trials, 400 trials were presented. A trial consisted of a series of three events: (1) a test portion in which the word "test" and a previously studied CVC appeared on the screen for $1.5 \mathrm{sec} ;(2)$ a dead period of $1 \mathrm{sec}$ in which $S$ was allowed to make his response; (3) a study portion in which the word "study" and a CVC-response pair were displayed for $2 \mathrm{sec}$.

On any trial a $\mathrm{k}$ item and a $\mathrm{u}$ item were equally likely to be presented for test. If a $\mathrm{k}$ item was presented in the test portion of a trial, it was also presented for study in the study portion of that same trial. However, once a $\mathbf{u}$ item was presented for test it was discarded, and a new $\mathbf{u}$ item was presented for study on that trial. Four $\mathbf{u}$ items were in the possible pool of test items at all times. Thus $\mathrm{S}$ had to remember at any time exactly four $\mathbf{u}$ items and two $\mathrm{k}$ items in order to respond at $100 \%$ correct. Results

The overall probability of a correct response on $u$ items showed a slight increase over the first three sessions and then remained relatively stable around a mean of .685 . The probability of a correct response for $k$ items was .998 over the entire experiment. For all subsequent data reported here, the first session and the first 25 trials of all other sessions were omitted to control for warm-up effects.

The lag of an item refers to the number of trials intervening between its study and subsequent test. If an item is presented on the study portion of a trial and is presented for test on the immediately following trial, the lag is 0 . If one complete trial intervenes between study and test, the lag is 1 , etc. Note that the test of an item always terminates the lag for that item. Hence an item cannot appear in its own lag. Also, u items are used only once in a session, while kitems are used repeatedly. Thus, all $u$ items in any lag will be different, but the same $\mathrm{k}$ item may reappear.

The data were analyzed for certain lags of interest. Figure 1 presents the observed probability of a correct response on a $\mathbf{u}$ item as a function of lag for three types of lags: (1) lag containing only k items; (2) lag contain- 


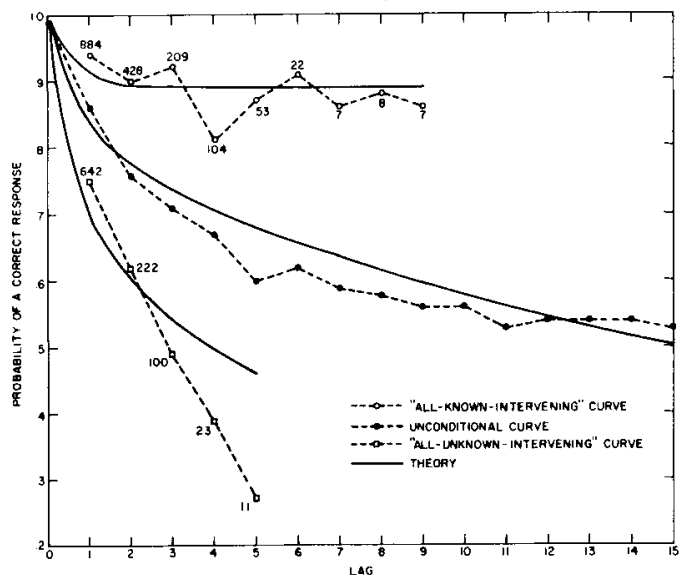

Fig. 1. Observed and theoretical probabilities of a correct response as a function of the lag between study and test.

ing only $u$ items; (3)- lag containing both $u$ and $k$ items. In this last curve the number of observations at each point decreases geometrically with parameter .875 from Lag 1 based on 1526 observations. The number of observations for all-k intervening and all-u intervening curves is appended to each point in Fig. 1.

\section{Discussion}

Atkinson \& Shiffrin (1967) proposed a model of memory and applied it to continuous presentation tasks like the one reported in the present study (Atkinson et al, 1967). The model postulates a rehearsal buffer of fixed size where items are processed and transcribed into long-term storage (LTS). Each item when presented for study has some probability $\alpha$ of entering the buffer and, if an item does enter, it randomly knocks out exactly one item currently in the buffer. Given these assumptions, the possibllity arises of different $\alpha$ values for different types of items. In particular, the application of the model to the present experiment assumes as a limiting case that $k$ items never enter the rehearsal buffer (i.e, $\alpha_{k}$ for $k$ items is 0 ), since $S$ has no reason to rehearse and process well-known items. Conversely, $\mathbf{u}$ items would have a nonzero probability $\alpha_{\mathbf{u}}$ of entering the buffer upon presentation.

Consider the curves presented in Fig. 1. A u item, say SOJ-7, is presented for study and with some probability $\alpha_{u}$ enters the rehearsal buffer from which recall is perfect. The only way SOJ-7 can be removed from the buffer before its test is to be knocked out by an ensuing item. Now, if all intervening items are $\mathrm{k}$ items, SOJ-7 cannot be knocked out of the buffer because $k$ items never enter the buffer. Hence recall for $\$ O J-7$ should be perfect at all lags, subject only to initially entering the buffer. Conversely, if only $u$ items intervene between the study and test of SOJ-7, the likelihood of replacing SOJ-7 in the buffer increases with lag, since every intervening item has some probability of entering the buffer and knocking SOJ-7 out. Somewhere in between should lie the unconditional curve; some of the items in the lag sequence will be $\mathrm{k}$ items and therefore will not disturb SOJ-7, but some will be $u$ items likely to replace SOJ-7.

In addition to $\alpha$, three other parameters are central to the model: the size of the buffer, $r$; the amount of information transferred from the buffer to LTS per trial, $\theta$; and the parameter governing the loss of information in LTS once an item has left the buffer, $T$. Using techniques similar to those described in Atkinson et al (1967), the buffer model was fit to the observed data in Fig. 1 using $\alpha_{k}$ set equal to one. The best fitting set of parameter values was $\alpha_{u}=.89, r=3, \theta=1.23$, and $T=.97$. The theoretical curves yielded by these parameter values are displayed in Fig. 1. It may readily be seen that while the fit is not perfect, the basic predicted patterns do hold. The greatest discrepancy between the model and the data is in the all-u intervening situation, with performance generally worse than predicted by the model. This discrepancy can perhaps be explained by assuming two values for the $\theta$ parameter, $\theta_{\mathrm{k}}$ applying on a trial when a $\mathrm{k}$ item is presented and $\theta_{\mathrm{u}}$ applying when a $u$ item is presented. Further, assume $\theta_{k}>$ $\theta_{u}$, i.e., more information transferred to LTS when a $k$ item is presented. Then, if $u$ items intervene, less information is transferred to LTS, resulting in depressed performance. A similar argument can be made for the $\tau$ parameter.

\section{References}

ATKINSON, R. C., \& SHIFFRIN, R. M. Human memory: a proposed system and its control processes. Tech. Rep. 110, Institute for Mathematical Studies in the Social Sciences, Stanford University, 1967. (To be published in K. W. Spence and J. T. Spence (Eds.) The Psychology of Learning and Motivation Advances in Research and Theory, Vol. 2, New York: Academic Press).

ATKINSON, R. C., BRELSFORD, J. W., \& SHIFFRIN, R. M. Multiprocess models for memory with applications to a continuous presentation task. J. Math. Psychol., 1967, 4, 277-300. 